\title{
Total- and semi-bare noble metal nanoparticles@silica core@shell catalysts for hydrogen generation by formic acid decomposition
}

\author{
Marco Santucci ${ }^{1} \cdot$ Maria Laura Ermini $^{1} \cdot$ Giulio Bresciani ${ }^{2} \cdot$ Andrea Griesi $^{1,3} \cdot$ Mauro Gemmi $^{1} \cdot$ Guido Pampaloni ${ }^{2}$. \\ Fabio Marchetti ${ }^{2} \cdot$ Valerio Voliani $^{1}$ (D)
}

Received: 27 November 2020 / Accepted: 27 December 2020 / Published online: 23 January 2021

(C) The Author(s) 2021

\begin{abstract}
Catalysts are involved in a number of established and emerging chemical processes as well as in environmental remediation and energy conversion. Nanoparticles (NPs) can offer several advantages over some conventional catalysts, such as higher efficiency and selectivity. Nowadays, versatile and scalable nanocatalysts that combine activity and stability are still lacking. Here, we report a comprehensive investigation on the production and characterization of hybrid nano-architectures bringing a partial or total bare surface together with their catalytic efficiency evaluation on, as a proof-of-concept, the formic acid decomposition reaction. In this regard, formic acid (FA) is a convenient and safe hydrogen carrier with appealing features for mobile applications, fuel cells technologies, petrochemical processes and energetic applications. Thus, the design of robust catalysts for FA dehydrogenation is strongly demanded. Due to this, we produced and evaluated nano-architectures with various equilibrium between the size-increase of the active part and the barer catalytic surface. Overall, this work paves the way for the development of new approaches for green energy storage and safe delivery.
\end{abstract}

Keywords Gold $\cdot$ Nanomaterials $\cdot$ Silica $\cdot$ Formic acid $\cdot$ Hydrogen

\section{Introduction}

One of the main fields in which engineered nanomaterials demonstrate their potential is in catalysis [1-4]. In particular, metal nanoparticles (NPs) are of special interest due to their increased catalytic activity with respect to bulk materials. On the other hand, their size may cause difficulties in handling and employing them in traditional industrial processes [5].

Marco Santucci and Maria Laura Ermini contributed equally

Fabio Marchetti

fabio.marchetti1974@unipi.it

Valerio Voliani

valerio.voliani@iit.it

1 Center for Nanotechnology Innovation@NEST, Istituto Italiano di Tecnologia, Piazza San Silvestro, 12-56126 Pisa, Italy

2 Department of Chemistry and Industrial Chemistry, University of Pisa, Via Moruzzi 13, 56124 Pisa, Italy

3 Department of Chemistry, Life Sciences and Environmental Sustainability, University of Parma, Parco Area delle Scienze 17/A, 43124 Parma, Italy
Indeed, NPs can be affected by sintering/stability and recovery issues [6]. An elegant strategy to both reduces NPs sintering while increasing their lifetime is the core@shell design, in which the active part is confined in the core and an external shell protect the catalyst [7]. This approach has the intrinsic advantage to increase the NPs recyclability and stability to coalescence due to the protective shell [8]. Moreover, the core@shell approach is usually versatile due to the easily tunable behaviors associated with the composition of the NPs $[7,9,10]$. On the other hand, the confinement of the active part reduces its accessibility and decreases the overall catalytic activity for some reactions [8]. In this context, investigations on the modulation of the catalytic activity associated to structural modifications of core@ shell NPs are pivotal to recognize the best structural equilibrium between the building blocks.

In this work, three core@shell nanocatalysts have been systematically investigated in order to maximize both the stability and catalytic activity over, as model reaction, the formic acid (FA) decomposition. FA is one of the major products from biomass processing and from a variety of chemical reactions based on methyl formate hydrolysis or $\mathrm{CO}_{2}$ hydrogenation [11]. Noticeably, FA decomposition is a virtuous, safe, cyclic, and convenient reaction for hydrogen delivery and 
generation. Indeed, hydrogen stored in FA can be released in situ by catalytic dehydrogenation [12]. It is worth to remember that the FA dehydration requires a $\mathrm{CO}$ resistant catalyst as gold because it shows better resistance to deactivation and poisoning with respect to $\mathrm{Pt}$ or $\mathrm{Ru}$ [13].

In this regard, we have employed as a first approach to the FA decomposition the passion fruit-like nano-architecture (NAs) [14]. NAs are silica nanocapsule comprising $3 \mathrm{~nm}$ ultrasmall gold NPs embedded and stabilized between two ionic polymers [15]. The passion fruit NAs are scalable, standardized, cheap and easily tunable during the synthesis by adapting the standard protocols $[14,16,17]$. From NAs, other two yolk-shell like nanostructures have been generated by an annealing process in air. The stabilizing agents (polymers) degradation and the annealing temperature enable the controlled metal sintering processes, with a consequent increase in NPs size. During the annealing treatments, nanomaterials with size-increased and bare active parts have been produced, the MultiCore $(m c)$ and SingleCore $(s c)$ NAs, thanks to polymers burning and ultrasmall metal NPs melting and re-condensation. Ideally, bare gold NPs comprised in NAs should present the maximum number of exposed active centers, resulting in a nanocatalyst that jointly combine efficiency with stability [18]. Here, the hybrid nano-architectures bringing a partial or total bare surface have been comprehensively characterized and their efficiency over the FA decomposition compared. This systematic work paves the way for the rational design of a low-cost versatile family of heterogeneous hybrid nanocatalysts for green energy storage and safe delivery of hydrogen.

\section{Results \& discussion}

\subsection{Multicore (mc) and Singlecore (sc) NAs synthesis}

A schematic representation for standard NAs production is reported in Fig. 1a [18]. The ultrasmall AuNPs (3 nm) are produced by a fast reduction of $\mathrm{HAuCl}_{4}$ (tetra chloroauric acid) by $\mathrm{NaBH}_{4}$ (sodium borohydride) in presence of PSS (poly(sodium 4-styrene sulfonate)). The solution turns instantly from bright yellow to dark red/brown and, in $10 \mathrm{~min}$, it becomes brilliant red due to the growth of $3 \mathrm{~nm}$ gold NPs. When PLL (poly-L-lysine) solution is added, NPs are assembled in spherical arrays by a controlled aggregation achieved by ionic interactions between PSS and PLL [17-19]. The polymer arrays are purified by centrifugation and employed in a Stöber reaction to form the silica shell. During the reaction, TEOS molecules tend to condense, crosslink and form a polysiloxane network covering the gold/polymers arrays, as reported by Cassano et al. [14]. After the Stöber reaction, the resulting standard NAs are centrifuged to discard the unreacted species. NAs are resuspended in EtOH by sonication and the nano-architectures bigger than about $150 \mathrm{~nm}$ removed by precipitation (Fig. S1). Then, collected particles are freeze-dried overnight. Usually, about $1.5 \mathrm{mg}$ of a shiny red powder is obtained from one-shot synthesis. This synthetic protocol can be actually scaled up to $10 \mathrm{X}$ in order to obtain up to $15 \mathrm{mg}$ of NAs. The product remains stable for 1 year if stored in the dark at $10{ }^{\circ} \mathrm{C}$ [18]. Remarkably, the reaction can be modulated in order to prepare NAs containing $\mathrm{Ag}, \mathrm{Pt}$ or mixed metals [14], and their raw production cost reduced by replacing PLL with PEI (polyethyleneimine) [19].

NAs can be converted in $m c$ NAs and $s c$ NAs by a controlled annealing treatment of the freeze-dried NAs powders. $m c \mathrm{NAs}$ and $s c \mathrm{NAs}$ comprise partially or totally bare gold NPs, which should maximize the number of active centres exposed on the metal surface [6]. Freeze-dried NAs appear as a ruby red powder (Fig. 1b). Interestingly, variations of the annealing treatments generate different products (Fig. $\mathrm{S} 2$ ). The $m c$ NAs are obtained by annealing NAs by the "single step-like" heating ramp (the temperature reaches $350^{\circ} \mathrm{C}$ in $15 \mathrm{~min}$ and is kept constant for $6 \mathrm{~h}$ ). After the thermal treatment, $m c$ NAs look as a black powder (Fig. 1b). The black color can be associated to the presence of unburned carbon compounds as a consequence of a not complete polymers oxidation [20]. Moreover, the unburned carbon compounds can act as physical stabilization agents for metal NPs that experience a partial sintering during the annealing treatment, which produces multinucleated NAs with metal cores of less than $10 \mathrm{~nm}$ in diameter (Table 1) [21].

The $s c$ NAs are obtained by NAs annealing following the "two step-like" heating ramp (Fig. S2). Briefly, the temperature reaches $200{ }^{\circ} \mathrm{C}$ in $15 \mathrm{~min}$, it is kept constant for $120 \mathrm{~min}$, increased up to $400{ }^{\circ} \mathrm{C}$ in $15 \mathrm{~min}$, still increased to $600{ }^{\circ} \mathrm{C}$ with a heating rate set at $100{ }^{\circ} \mathrm{C} \mathrm{h}^{-1}$, and maintained at $600{ }^{\circ} \mathrm{C}$ for $105 \mathrm{~min}$. After the thermal treatment, $s c$ NAs are collected as a pink powder (Fig. 1b). The $s c$ NAs pink color can be associated to the metal NPs size that changed during the treatment and to the changed dielectric environment [22]. Indeed, scNAs are composed by a single and bare gold nanoparticle of about $20 \mathrm{~nm}$ inside the silica capsule (Table 1).

\section{2 mcNAs and scNAs characterization}

The three species of NAs have been comprehensively characterized by using TEM imaging for the morphologic investigation (Fig. 1b); FTIR (Fourier Transform InfraRed) spectroscopy (Fig. 2a) and EELS (Electron Energy Loss Spectroscopy) (Fig. 2b) to check polymers behavior induced by the annealing; ICP-MS (Inductively Coupled Plasma Mass Spectrometry) for elemental quantitative analysis (Table 1); powder XRD (X-Ray Diffraction) (Fig. 2b) for NAs phase identification and characterization. 
a

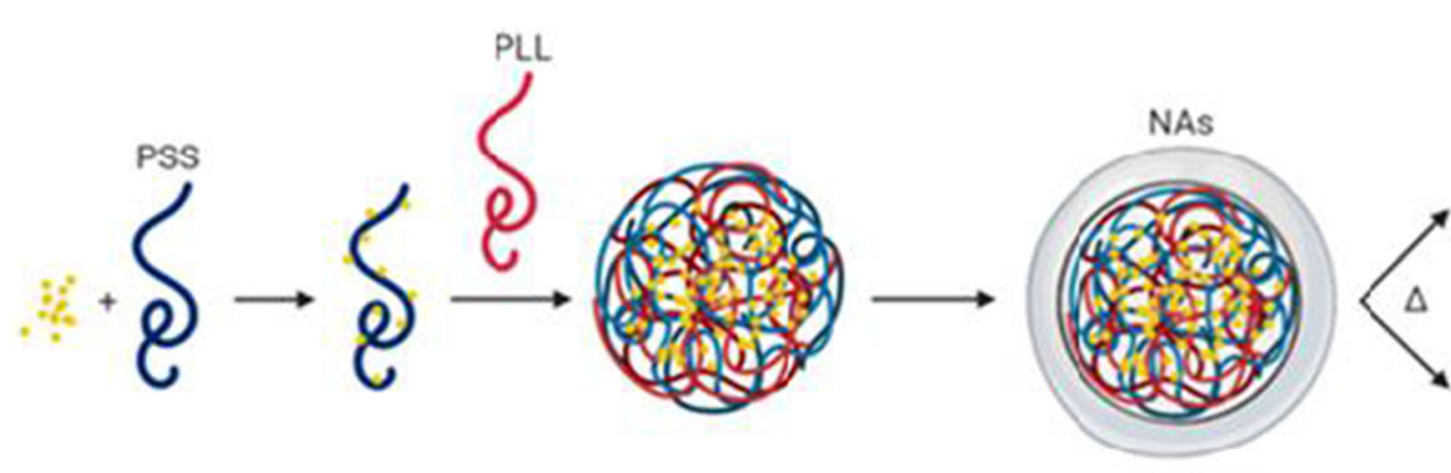

b
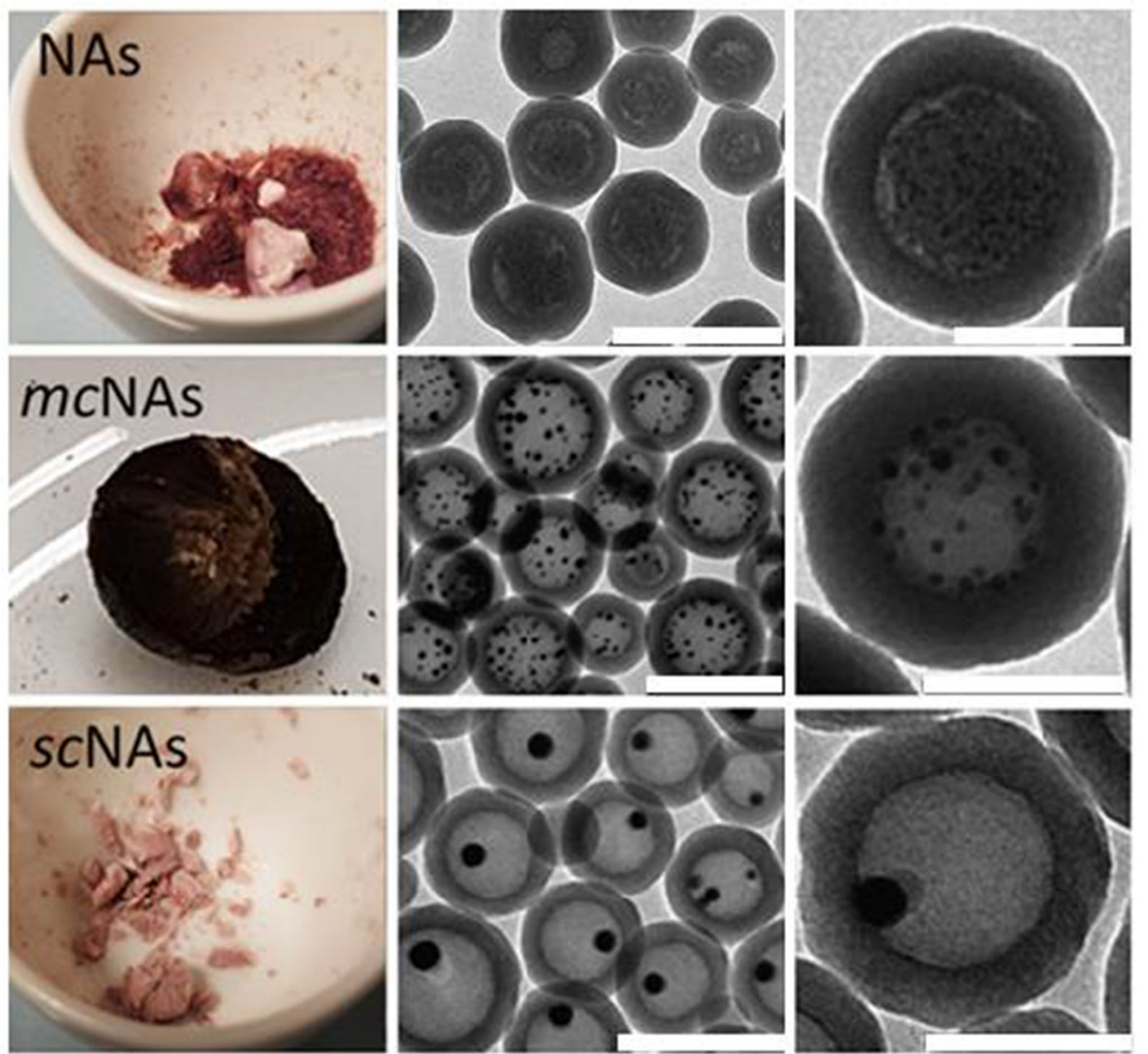

Fig. 1 a General synthesis scheme for the composition of NAs. $3 \mathrm{~nm}$ ultrasmall gold nanoparticles are stabilized by poly(sodium 4-styrene sulfonate) and assembled in polymer arrays by employing poly(L-lysine). Then, the templates are covered by a silica shell [18]. NAs can be further converted in multicore $(\mathrm{mc})$ or singlecore (sc) NAs by an annealing

FTIR spectroscopy provides molecular details about functional groups comprised in the nano-architectures. The FTIR spectra of NAs, PLL and PSS are reported together with their peak's assignment in Fig.S3 and process. $m c$ NAs and $s c$ NAs comprise partially or totally bare gold NPs. b In each row are reported a photo of the NAs powders (left), and two bright field TEM images at different magnifications, a larger area at lower magnification (center) and a higher magnification taken on one single particle (right). Scalebars are $200 \mathrm{~nm}$ (center) and $100 \mathrm{~nm}$ (right)

Table S4. The FTIR spectra of NAs, $m c$ NAs and $s c$ NAs are reported in Fig.2a.

In the NAs spectrum, two distinct sets of relevant peaks can be observed. The first, it is the $\mathrm{C}=\mathrm{O}$ amide group stretching 
Table 1 Average diameters of NAs (first column), average thickness of the silica shell (second column), and average diameter of gold nanoparticle(s) comprised in the nanocapsules (third column). Analyses performed on at least 50 NAs collected by TEM. Quantitative ICP-MS analyses of gold comprised in NAs (last column). The Au weight percentage is referred to the ratio between the weight of Au contained in the NAs and the total weight of the freeze-dried samples

\begin{tabular}{lllll}
\hline & Silica capsule diameter (nm) & Shell thickness (nm) & AuNPs size (nm) & Au weight \% \\
\hline NAs & $110 \pm 19$ & $33 \pm 4$ & $2.8 \pm 0.5$ & $7.4 \pm 0.6 \%$ \\
mcNAs & $122 \pm 21$ & $34 \pm 3$ & $8 \pm 2$ & $8 \pm 1 \%$ \\
scNAs & $123 \pm 34$ & $25 \pm 3$ & $18 \pm 9$ & $7.3 \pm 0.7 \%$ \\
\hline
\end{tabular}

peaked at $1650 \mathrm{~cm}^{-1}$, which derives from PLL, combined with the water bending peak at $1640 \mathrm{~cm}^{-1}$ [23]. The second, it is the Si-O stretching band between 1500 and $800 \mathrm{~cm}^{-1}$ associated to the silica shell, which is very intense respect to the amide signal. This set is formed by three main peaks, which are at $1157,1054,940 \mathrm{~cm}^{-1}[23,24]$. The PSS peaks are weak and masked in the NAs spectrum. Indeed, the PSS degradation cannot be directly confirmed from the IR spectra in $m c$ NAs and $s c$ NAs. On the other hand, the $\mathrm{C}=\mathrm{O}$ peak decrease gradually in intensity from NAs to $m c$ NAs and $s c$ NAs. This result may suggest the gradual PLL degradation promoted by the annealing process but cannot be confirmed because of the presence of the water bending peak jointly combined with the low intensity variation. The stretching mode peaked at $1157 \mathrm{~cm}^{-1}$ for the Si-O bands decreases gradually in intensity up to become a shoulder. Also, the $940 \mathrm{~cm}^{-1}$ peak gradually decreases in intensity from NAs to $s c$ NAs. These spectral changes can be associated to structural modifications of the silica shell promoted by the annealing process, such as a gradual silica crosslink and reorganization [25].

The EELS spectrum of $s c$ NAs has been collected to further investigate the polymers oxidation and partial escape during the annealing process (Fig. 2b). This technique measures the energy distribution of electrons that have interacted with the sample and their energy loss due to inelastic scattering [26]. Thus, elemental analysis can be performed by detecting in the ELLS spectrum the energy loss peaks corresponding to the ionization energies of the different elements (ionization edges) [26]. This technique is particularly efficient for light elements. At $165 \mathrm{eV}$, the sulfur L edge peak is weak but present, highlighting a possible presence of $\mathrm{S}$ species in $s c \mathrm{NAs}$. This finding may be associated to a partial escape of sulfur produced by the degradation of the PSS sulfonate groups due to its strong affinity with gold [21]. Instead, the nitrogen $\mathrm{K}$ edge peak at $400 \mathrm{eV}$ is completely absent. By combining both the EELS and FTIR results, the PLL degradation and its products escape from $s c$ NAs is confirmed. The carbon signal in the spectrum is mainly due to the carbon film of the sample holder grid, while the oxygen signal comes from the silica shell.

Powder XRD has been employed to collect qualitative structural information about NAs, $m c \mathrm{NAs}$ and $s c \mathrm{NAs}$ and their components. Their PXRD patterns are reported in Fig. 2c. NAs pattern (green) display the silica characteristic peak at $2 \theta$ value of $22.74^{\circ}$, which confirms the amorphous nature of the silica shell [27]. Four broad peaks are present at $2 \theta$ values of $38.49^{\circ}, 44.06^{\circ}, 65.51^{\circ}$ and $78.49^{\circ}$, which can be indexed as the $111,200,220$ and 311 reflections of face centred cubic (FCC) gold. The broadness of these peaks reveals the nanometric size of the ultrasmall AuNPs [28]. Interestingly, the silica peak of $m c \mathrm{NAs}$ and $s c \mathrm{NAs}$ experiences a shift up to $21.46^{\circ}$. Taken together with the FTIR spectra, this result confirms the silica shell reorganization induced by the annealing process $[25,29]$. This sharpening allows to see in $m c$ NAs and $s c$ NAs the weak 222 reflection of gold FCC lattice at $81.7^{\circ}$ [30, 31]. The gradual gold peaks sharpening, from NAs to $m c \mathrm{NAs}$ and $s c \mathrm{NAs}$, is related to the increasing size of the coherent crystalline domains of gold during the annealing, which tend to grow up to about $20 \mathrm{~nm}$ in the $s c$ NAs [32].

\subsection{Formic acid catalytic decomposition}

Formic Acid ( $\mathrm{HCOOH}$ or FA) is a convenient and safe $\mathrm{H}_{2}$ carrier useful in mobile applications, fuel cells technologies, petrochemical processes, and energy delivery and conversion [33]. On this hand, FA is considered a good candidate to satisfy the increasing demands of high purity $\mathrm{H}_{2}$ [34, 35]. In this context, FA controlled, efficient and selective dehydrogenation by a robust catalyst is strongly demanded [5]. Pt, Ir, Pd, $\mathrm{Ru}, \mathrm{Rh}$ catalyst are not suitable choices for this reaction due to the presence of an equilibrium between FA dehydrogenation and dehydration. FA dehydration tends to form $\mathrm{H}_{2} \mathrm{O}+\mathrm{CO}$. $\mathrm{CO}$ levels at temperature close to $100{ }^{\circ} \mathrm{C}$ become relevant and usually deactivate the catalyst [33].

Several groups have investigated the efficiency of homogeneous or heterogeneous catalysts for FA decomposition. Coffey et al. describe a pioneering approach by employing iridium phosphine complex for the decomposition of FA in acetic acid at about $110^{\circ} \mathrm{C}$ [36]. Furthermore, many transition metal complexes have been tested in this model reaction, such as [Ru2( $\mu-\mathrm{CO})(\mathrm{CO}) 4(\mu-\mathrm{dppm}) 2]$ [37], [RhCp*(bpy)Cl]Cl [38], $\left[\mathrm{Fe}\left(\mathrm{BF}_{4}\right)_{2}\right] \times 6 \mathrm{H}_{2} \mathrm{O}$ [39], [IrCp*(4,4'-hydroxy-2,2'bipyridine)] [40]. Generally, the main goal of these 


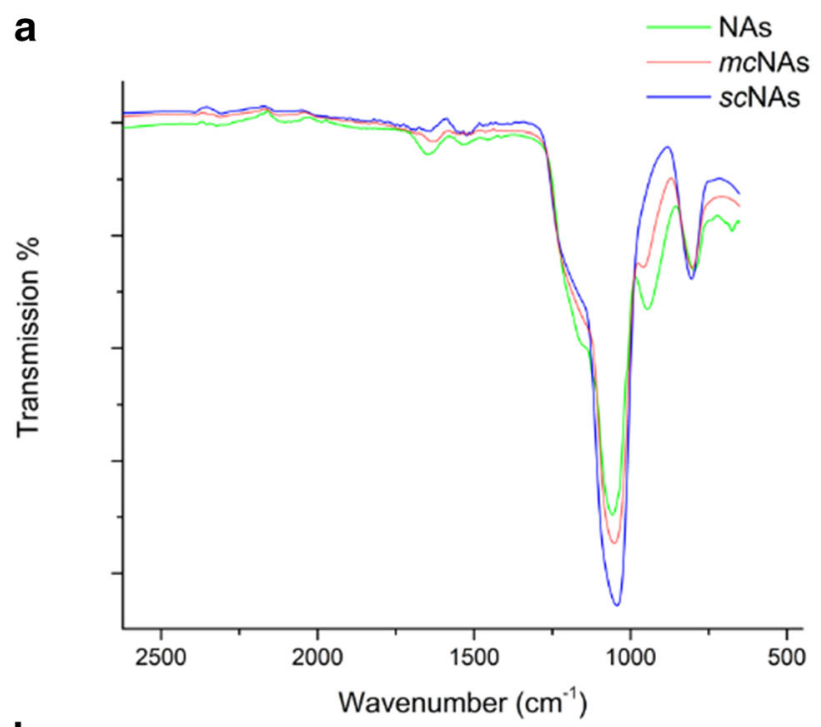

b

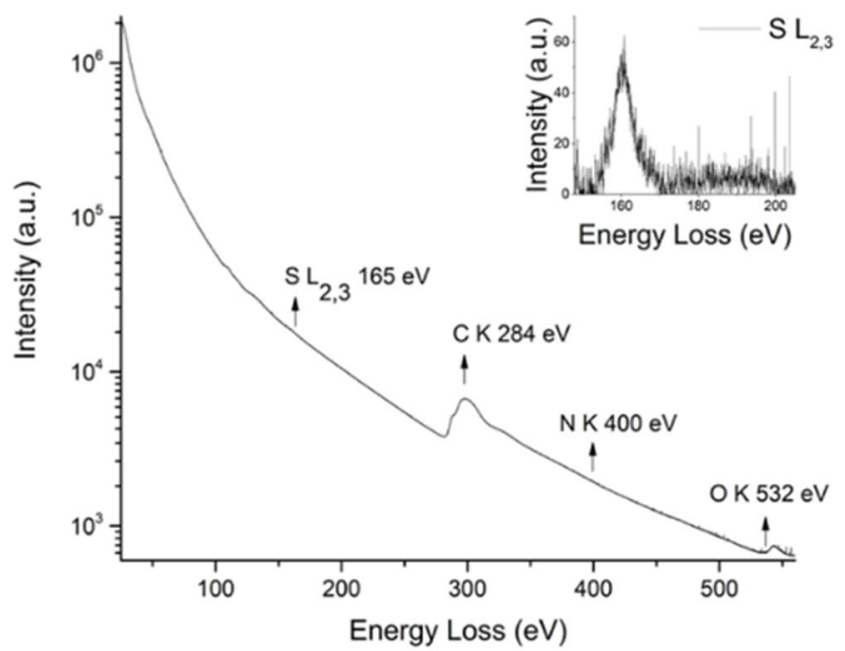

C

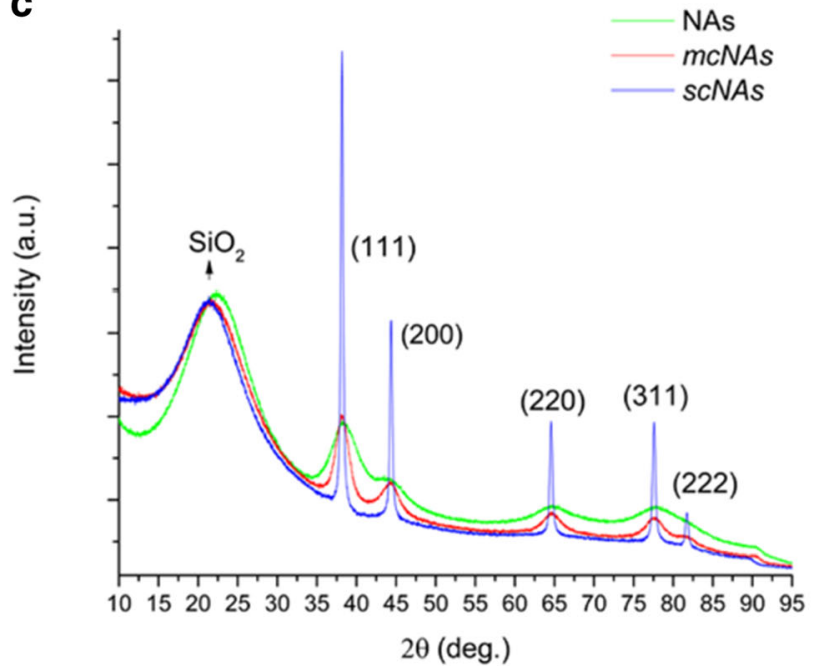

Fig. 2 a FTIR spectra of NAs (green), $m c$ (red) and $s c$ (blue) NAs. Spectrum resolution about $1.9 \mathrm{~cm}^{-1}$. b scNAs EELS spectrum. Peak labels are superimposed with the spectrum and assigned by comparison with the EELS atlas database. C) NAs (green), $m c$ (red) and $s c$ NAs (blue) superimposed powder XRD patterns investigations was to achieve the higher turnover frequency at the lowest temperature [33]. Concerning heterogeneous catalysts, mono- and bi-metallic catalysts have been the most widely investigated for FA decomposition, among which $\mathrm{PdAu} / \mathrm{C}-\mathrm{CeO}_{2}$ [41], $\mathrm{Pd} / \mathrm{C}$ [42], Ag@Pd/C [43]. Ojeda et al. [44] reported that finely dispersed AuNPs supported on $\mathrm{Al}_{2} \mathrm{O}_{3}$ exhibit high activity toward FA dehydrogenation. Au also exhibits a catalytic activity in the Water Gas Shift (WGS) reaction. Thanks to WGS, CO levels can be minimized by the same gold catalyst by its in situ conversion to $\mathrm{H}_{2}$ and $\mathrm{CO}_{2}$. Overall, AuNPs are promising catalysts for this reaction. As reported by Bi et al. [34], the formic acid / triethylamine (FA/ $\mathrm{NEt}_{3}$ ) adduct decomposition is a suitable model reaction to investigate the catalytic activity and stability of nanomaterials. $\mathrm{NEt}_{3}$ is usually employed as co-catalyst to improve the reaction yield since it forms a quasi-ionic adduct that promotes the dehydrogenation process [45]. NAs, $m c \mathrm{NAs}$, and $s c$ NAs catalytic activity has been evaluated on $\mathrm{FA} / \mathrm{NEt}_{3}$ adduct by employing the "water displacement method" (Fig. 3A). This experimental approach is appropriate for reaction in which low water soluble gas mixtures are generated [46].

In Fig. 3B and Fig. S5 the FA catalytic decomposition performances of NAs, $m c$ NAs and $s c$ NAs are reported. Catalysts are dispersed in $\mathrm{FA} / \mathrm{NEt}_{3}$ pure adduct $\left(\mathrm{FA} / \mathrm{NEt}_{3}\right.$ 1:1 mol ratio) and tested at $60{ }^{\circ} \mathrm{C}$, monitoring the evolved gas as a function of reaction time. From the kinetic data, all the tested catalysts exhibit an improved gas evolution respect to the control. Usually, the catalytic performance of nanomaterials decreases dramatically with the $\mathrm{S} / \mathrm{V}$ ratio [6]. In this case, the reaction rate decrease is relatively mild for $m c \mathrm{NAs}$ and $s c \mathrm{NAs}$. Ideally, a partially or totally bare catalyst, respectively $m c \mathrm{NAs}$ and $s c \mathrm{NAs}$, tends to expose the maximum number of active sites on the catalyst surface [47]. Thus, the two opposite effects are buffered and both the $m c$ NAs and $s c$ NAs still maintain some catalytic features.

TEM imaging has been performed in order to investigate potential structural alterations induced by the catalytic tests (Fig. 3C). Frequently, catalysts experience morphological modifications as sintering, which negatively affect the catalytic activity and recycling $[48,49]$. On this hand, NAs are designed to avoid these effects and improve recyclability and stability [50]. In particular, the hollow silica capsules efficiently protect the core catalyst from coalescence phenomena by simply acting as a physical shield [7]. NAs, $m c \mathrm{NAs}$, and $s c$ NAs do not display significant morphologic changes after the catalytic tests. In particular, no gold leakage nor NAs shell damage have been observed. Evidences connected with sintering phenomena, such as coalescence and Ostwald ripening, are not found. This evidence confirms that the silica shell can improve the lifetime of the catalyst in this reaction conditions. 
a

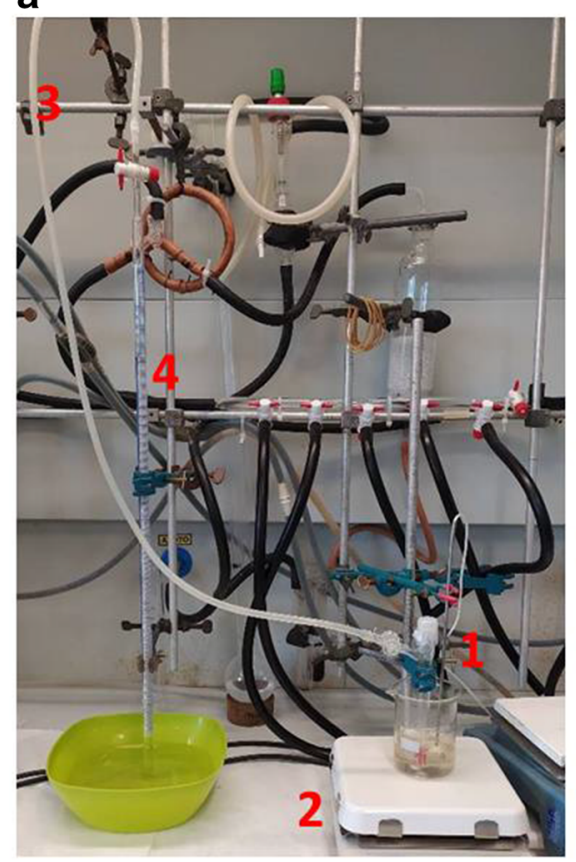

C

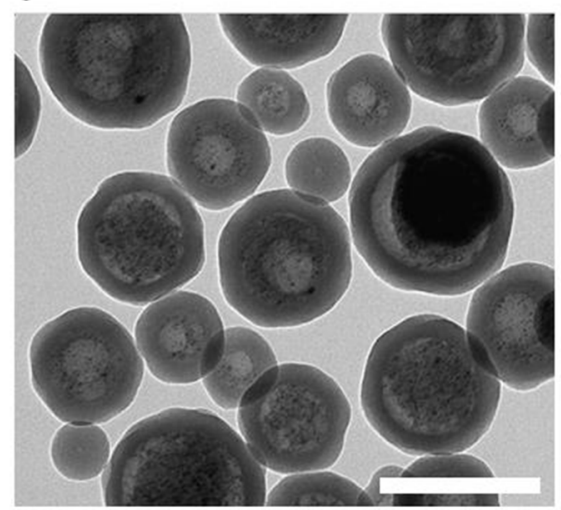

b
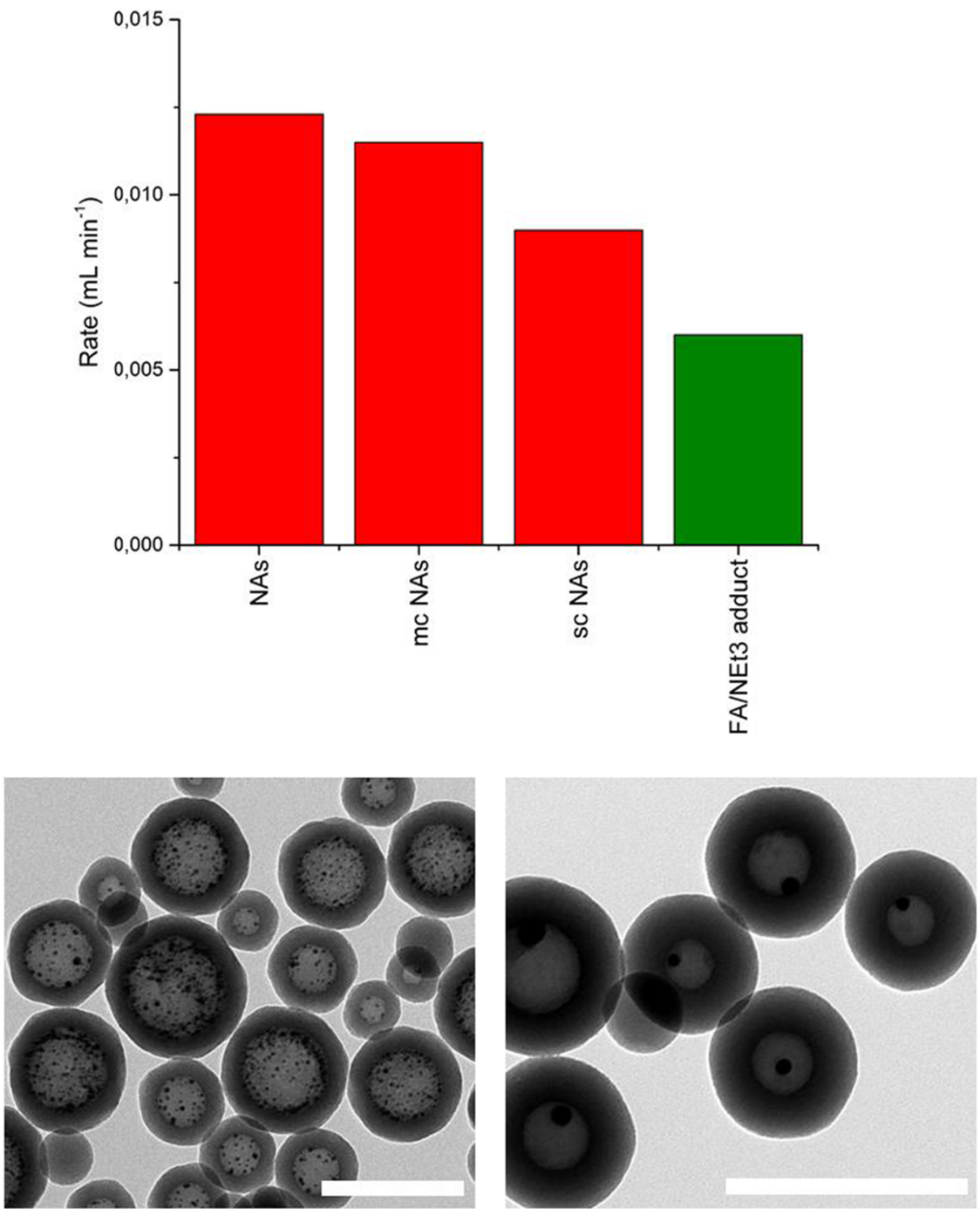

Fig. 3 a Experimental setup used for the FA/NEt 3 adduct catalytic decomposition. As reactor, a Schlenk tube (1) mounted on a stirrer/ thermal heater was used (2). Evolved gasses in the Schlenk reach by a tube (3) the volume measurement apparatus (4), which is an upside down $25 \mathrm{~mL}$ burette filled with oversaturated salt solution. The gas mixture, which escapes from the reactor, reaches the burette and displaces the solution column for a volume equal to the reaction evolved gas. b
Reaction rate of NAs, $m c$ NAs, and $s c$ NAs. In red the samples, and in green, as control, the reaction rate of the pure $\mathrm{FA} / \mathrm{NEt}_{3}$ adduct decomposition. Reaction conditions: $5 \mathrm{mg}$ of catalyst is tested with $1.2 \mathrm{~mL}$ scale of $\mathrm{FA} / \mathrm{NEt}_{3}$ pure adducts $(1: 1 \mathrm{~mol}$ ratio $)$ at $60^{\circ} \mathrm{C}$. c Post catalysis NAs TEM morphologic investigation. Left, center and right are TEM bright field images of NAs, $m c$ NAs and $s c$ NAs, respectively. Scale bars $200 \mathrm{~nm}$

\section{Conclusions}

In summary, we have demonstrated the controlled composition of a family of versatile nano-architectures comprising a partially or completely bare active part. Besides their comprehensive characterizations, we compared their activity toward the FA decomposition for the in situ generation of hydrogen. Interestingly, we recognized a decrease in the catalytic activity as a function of the annealing temperature, that is associated to the equilibrium between the size-increase of the active part and the barer catalytic surface, confirming the rationally design of nanocatalysts is an essential pre-requisite.

\section{Materials and methods}

\subsection{Nanoarchitectures (NAs) production}

The following protocol was standardized for the production of $1.5 \mathrm{mg}$ NAs in about $4 \mathrm{~h}$. The protocol can be scaled-up to $15 \mathrm{mg}$ [18].

\subsubsection{Gold nanoarchitecture}

Synthesis of Ultrasmall gold nanoparticles In a rounded flask with $20 \mathrm{~mL}$ of milliQ water, $10 \mu \mathrm{L}$ of $30 \%$ PSS (poly(sodium 
4-styrene sulfonate)) solution are added during vigorous stirring. $200 \mu \mathrm{L}$ of $10 \mathrm{mg} / \mathrm{mL} \mathrm{HAuCl}_{4}$ (tetra chloroauric acid) are added to the solution pot. In a single fast shot, $200 \mu \mathrm{L}$ of $8 \mathrm{mg} /$ $\mathrm{mL} \mathrm{NaBH}_{4}$ (sodium borohydride) solution are added. The solution turns instantly from bright yellow to dark red/brown and in $10 \mathrm{~min}$ it becomes a brilliant red.

Synthesis of gold nanoparticles arrays At very low stirring rate, $75 \mu \mathrm{L}$ of $40 \mathrm{mg} / \mathrm{mL}$ PLL (poly-L-lysine) solution are added. After $20 \mathrm{~min}$ of gentle stirring, the solution is centrifuged at $14000 \mathrm{rpm}$ for $3 \mathrm{~min}$. The supernatant is discarded and $2 \mathrm{~mL}$ of milliQ water is added to the centrifuge tube to resuspend the precipitate helped by a gentle sonication. All the precipitate that did not resuspend is discarded.

Synthesis of NAs The $2 \mathrm{~mL}$ nanoarrays solution is divided in two tubes, $1 \mathrm{~mL}$ each, to perform the Stöber reaction where silica shell is formed. In each tube, $35 \mathrm{~mL}$ of EtOH (ethanol) is mixed with $1.2 \mathrm{~mL}$ of $30 \% \mathrm{NH}_{4} \mathrm{OH}$ (ammonia solution) and $20 \mu \mathrm{L}$ of TEOS (tetraethyl orthosilicate).

These tubes are closed and gently shaken for $3 \mathrm{~h}$ on a tilting plate. The tubes are centrifuged for $30 \mathrm{~min}$ at $4000 \mathrm{rpm}$ to discard the supernatant. The precipitated NAs are resuspended in $4 \mathrm{~mL}$ of EtOH by sonication and they are washed two times in EtOH. After a short spin centrifugation of $15 \mathrm{~s}$ (reaching $14 \mathrm{k} \mathrm{rpm}$ ), NAs bigger than about $150 \mathrm{~nm}$ precipitate, and the supernatant contains monodisperse NAs. The supernatant collected particles are freeze-dried overnight. Usually, about $1.5 \mathrm{mg}$ of a brilliant red powder is obtained from one-shot synthesis. The product remains stable for 1 year stored in the dark at $10^{\circ} \mathrm{C}$ [14]. This synthetic protocol can be scaled up to $10 \mathrm{X}$ reactant quantities to obtain up to $15 \mathrm{mg}$ of NAs with a single synthesis.

\subsection{Multicore (mcNAs) and singlecore (scNAs) nanoarchitectures production}

Freeze dried NAs are transferred in a crucible pot for high temperature and annealed in a standard furnace following the reported sequence:

For $m c \mathrm{NAs}$, in $15 \mathrm{~min}$ the temperature of $350^{\circ} \mathrm{C}$ is reached and kept constant for $6 \mathrm{~h}$.

For $s c$ NAs, in $15 \mathrm{~min}$ the temperature of $200^{\circ} \mathrm{C}$ is reached and kept constant. At $135 \mathrm{~min}$, the temperature is raised up to $400{ }^{\circ} \mathrm{C}$ in $15 \mathrm{~min}$. Reached $400{ }^{\circ} \mathrm{C}$, the heating rate is set to $100{ }^{\circ} \mathrm{C} \mathrm{h}^{-1}$ and maintained for $2 \mathrm{~h}$. Reached $600{ }^{\circ} \mathrm{C}$, the temperature is maintained constant for $105 \mathrm{~min}$.

It is recommended to wait the cool down of the furnace before removing the samples. The resulting powder undergoes a color change turning black for $m c$ and shiny pink for $s c$ NAs. Samples are recovered from the crucible by dispersing it in $1 \mathrm{~mL}$ of $\mathrm{EtOH}$.

\subsubsection{Transmission Electron microscopy (TEM)}

TEM observations of nanoparticles are carried out on a ZEISS Libra 120 TEM operating at an accelerating voltage of $120 \mathrm{kV}$, equipped with an in-column omega filter to perform energy filtered imaging and EELS analysis. The colloidal solutions are deposited on 300-mesh carbon-coated copper grids and observed after at least $5 \mathrm{~h}$ from the deposition.

\subsubsection{FT-IR spectroscopy}

Infrared spectra are recorded using a Diamond ATR sampling block for a Cary 630 FTIR (Agilent Technologies, Santa Clara, CA, USA). The spectra of polymers are obtained from around $10 \mu \mathrm{L}$ of liquid samples (50\% in water). NAs spectra are recorded with around $100 \mu \mathrm{g}$ of freeze-dried samples.

\subsubsection{ICP-MS analyses}

Nanoparticles are dissolved in $1 \mathrm{~mL}$ ICP-MS grade $\mathrm{HNO}_{3}$ and digested by microwave irradiation $\left(200{ }^{\circ} \mathrm{C} / 15 \mathrm{~min}\right)$ in borosilicate glass vessels. The resulting solution is diluted to $10 \mathrm{~mL}$ with ICP-MS grade water, and content of gold was determined by ICP-MS analysis against a standard calibration curve.

\subsubsection{PXRD analyses}

NAs are characterized through powder XRD by recording their patterns on a STOE Stadi P X-ray powder diffractometer working in Debye-Scherrer geometry equipped with $\mathrm{Cu}-\mathrm{K}_{\alpha 1}$ radiation $(\lambda=1.5406 \AA$ ), a Ge (111) Johansson monochromator and a MYTHEN2 $1 \mathrm{~K}$ detector from Dectris. The XRD patterns have been collected in a $2 \theta$ range of $10-95^{\circ}$, with a step of $0.03^{\circ}$ and a time/step of $6 \mathrm{~s}$. Samples are gently grounded into a fine powder with pestle and mortar and transferred in a $0.8 \mathrm{~mm}$ silica capillary, which is placed on the diffraction holders.

\subsection{Catalytic tests}

Liquid-phase formic acid decomposition is conducted in a $5 \mathrm{~mL}$ Schlenk reactor. The reactor is placed in a paraffin oil bath and a heater/magnetic stirrer is sets at $1.1 \mathrm{k} \mathrm{rpm}$ of stirring rate and a temperature of $60{ }^{\circ} \mathrm{C}$. The Schlenk reactor is connected to a displacement volume meter thanks to a tube. The typical experimental procedure was the following. $5 \mathrm{mg}$ of dried nano-architectures (around $400 \mu \mathrm{g}$ in gold) are dispersed in $0.5 \mathrm{~mL}$ of pure FA helped by a gentle sonication. The adduct $\mathrm{FA} / \mathrm{NEt}_{3}$ is formed (FA / $\mathrm{NEt}_{3}$ 1:1 mol ratio) and transferred in the Schlenk reactor, which is already thermoset at $60{ }^{\circ} \mathrm{C}$, during high stirring rate. The gas evolution is monitored in an upside down $25 \mathrm{~mL}$ burette filled with 
oversaturated salt solution. This method is also called water displacement method. Experimental data, which are curves of evolved gas volume $(\mathrm{mL})$ vs time $(\mathrm{min})$, are obtained by a single test, linearly fitted and intercept corrected to extract the rate slope expressed in $\mathrm{mL}$ of evolved gas versus time $(\mathrm{mL} / \mathrm{min})$.

Supplementary Information The online version contains supplementary material available at https://doi.org/10.1007/s42247-020-00160-8.

Author contributions M.S. and M.L.E. nano-architectures synthesis and characterizations; A.G., and M.G. XRD and EELS data collection and interpretation; M.S. and G.B catalytic tests; G.P., F.M. and V.V. design, coordination of the project and funding. All Authors have discussed the data and contributed to writing the manuscript.

Funding Open Access funding provided by Istituto Italiano di Tecnologia.

\section{Compliance with ethical standards}

Conflict of interest The authors declare no conflict of interest.

Open Access This article is licensed under a Creative Commons Attribution 4.0 International License, which permits use, sharing, adaptation, distribution and reproduction in any medium or format, as long as you give appropriate credit to the original author(s) and the source, provide a link to the Creative Commons licence, and indicate if changes were made. The images or other third party material in this article are included in the article's Creative Commons licence, unless indicated otherwise in a credit line to the material. If material is not included in the article's Creative Commons licence and your intended use is not permitted by statutory regulation or exceeds the permitted use, you will need to obtain permission directly from the copyright holder. To view a copy of this licence, visit http://creativecommons.org/licenses/by/4.0/.

\section{References}

1. D. Astruc, Introduction: Nanoparticles in Catalysis. Chem. Rev. 120, 461-463 (2020)

2. N. Lakshmana Reddy, V. Navakoteswara Rao, M. Mamatha Kumari, R.R. Kakarla, P. Ravi, M. Sathish, M. Karthik, S. Muthukonda Venkatakrishnan, Inamuddin, Nanostructured semiconducting materials for efficient hydrogen generation. Environ. Chem. Lett. 16, 765-796 (2018)

3. M. Zarezadeh Mehrizi, J. Abdi, M. Rezakazemi, E. Salehi, A review on recent advances in hollow spheres for hydrogen storage. Int. J. Hydrog. Energy 45, 17583-17604 (2020)

4. M.A. Ahmed, N.M. Abdelbar, A.A. Mohamed, Molecular imprinted chitosan- $\mathrm{TiO}_{2}$ nanocomposite for the selective removal of Rose Bengal from wastewater. Int. J. Biol. Macromol. 107, 1046-1053 (2018)

5. T. Hayashi, K. Tanaka, M. Haruta, Selective Vapor-Phase Epoxidation of Propylene over $\mathrm{Au} / \mathrm{TiO}_{2}$ Catalysts in the Presence of Oxygen and Hydrogen. J. Catal. 178, 566-575 (1998)

6. A. Alshammari, V. N. Kalevaru, Supported Gold Nanoparticles as Promising Catalysts. Catal. Appl. Nano-Gold Catal. (2016)

7. X.-Q. Cao, J. Zhou, S. Li, G.-W. Qin, Ultra-stable metal nanocatalyst synthesis strategy: a perspective. Rare Metals 39, 113$130(2020)$
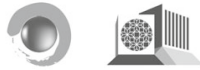
QATAR UNIVERSITY
8. Q. Zhang, I. Lee, J.B. Joo, F. Zaera, Y. Yin, Core-Shell Nanostructured Catalysts. Acc. Chem. Res. 46, 1816-1824 (2013)

9. V. Sunny, D. Sakthi Kumar, Y. Yoshida, M. Makarewicz, W. Tabiś, M.R. Anantharaman, Synthesis and properties of highly stable nickel/carbon core/shell nanostructures. Carbon 48, 1643-1651 (2010)

10. J. Zhou, F. Ren, S. Zhang, W. Wu, X. Xiao, Y. Liu, C. Jiang, $\mathrm{SiO}_{2}-$ $\mathrm{Ag}-\mathrm{SiO}_{2}-\mathrm{TiO}_{2}$ multi-shell structures: plasmon enhanced photocatalysts with wide-spectral-response. J. Mater. Chem. A 1, $13128(2013)$

11. C. Federsel, R. Jackstell, M. Beller, State-of-the-Art Catalysts for Hydrogenation of Carbon Dioxide. Angew. Chem. Int. Ed. 49, 6254-6257 (2010)

12. F. Valentini, V. Kozell, C. Petrucci, A. Marrocchi, Y. Gu, D. Gelman, L. Vaccaro, Formic acid, a biomass-derived source of energy and hydrogen for biomass upgrading. Energy Environ. Sci. 12, 2646-2664 (2019)

13. S.A.C. Carabineiro, Supported Gold Nanoparticles as Catalysts for the Oxidation of Alcohols and Alkanes. Front. Chem. 7 (2019)

14. D. Cassano, J. David, S. Luin, V. Voliani, Passion fruit-like nanoarchitectures: a general synthesis route. Sci. Rep. 7, 43795 (2017)

15. D. Cassano, M. Summa, S. Pocoví-Martínez, A.-K. Mapanao, T. Catelani, R. Bertorelli, V. Voliani, Biodegradable Ultrasmall-inNano Gold Architectures: Mid-Period In Vivo Distribution and Excretion Assessment. Part. Part. Syst. Charact. 36, 1800464 (2019)

16. A.K. Mapanao, M. Santi, V. Voliani, Combined chemophotothermal treatment of three-dimensional head and neck squamous cell carcinomas by gold nano-architectures. J. Colloid Interface Sci. 582, 1003-1011 (2021)

17. S. Pocoví-Martínez, D. Cassano, V. Voliani, Naked Nanoparticles in Silica Nanocapsules: A Versatile Family of Nanorattle Catalysts. ACS Appl. Nano Mater. 1, 1836 (2018)

18. D. Cassano, D.R. Martir, G. Signore, V. Piazza, V. Voliani, Biodegradable hollow silica nanospheres containing gold nanoparticle arrays. Chem. Commun. 51, 9939-9941 (2015)

19. G. Giannone, M. Santi, M.L. Ermini, D. Cassano, V. Voliani, A Cost-Effective Approach for Non-Persistent Gold NanoArchitectures Production. Nanomaterials 10, 1600 (2020)

20. G. M. Jenkins, J. Alan, K. Kawamura, Polymeric Carbons: Carbon Fibre, Glass and Char (Cambridge University Press, 1976)

21. L.M. Rossi, J.L. Fiorio, M.A.S. Garcia, C.P. Ferraz, The role and fate of capping ligands in colloidally prepared metal nanoparticle catalysts. Dalton Trans. 47, 5889-5915 (2018)

22. A. Derkachova, K. Kolwas, I. Demchenko, Dielectric Function for Gold in Plasmonics Applications: Size Dependence of Plasmon Resonance Frequencies and Damping Rates for Nanospheres. Plasmonics 11, 941-951 (2016)

23. P. Larkin, in Infrared and Raman Spectroscopy. IR and Raman Spectra-Structure Correlations (Elsevier, Oxford, 2011), pp. 73115

24. A.S. Khan, H. Khalid, Z. Sarfraz, M. Khan, J. Iqbal, N. Muhammad, M.A. Fareed, I.U. Rehman, Vibrational spectroscopy of selective dental restorative materials. Appl. Spectrosc. Rev. 52, 507-540 (2017)

25. A. Alattar, W. Twej, M. Drexlerp, F. Alamgir, Study the Annealing Temperature Influence on the Mesoporous Silica Aerogel Properties via Supercritical Drying. Int. J. Innov. Sci. Eng. Technol. 3, 6 (2016)

26. R.F. Egerton, Electron energy-loss spectroscopy in the TEM. Rep. Prog. Phys. 72, 016502 (2008)

27. N.Q. Yin, P. Wu, T.H. Yang, M. Wang, Preparation and study of a mesoporous silica-coated $\mathrm{Fe}_{3} \mathrm{O}_{4}$ photothermal nanoprobe. RSC Adv. 7, 9123-9129 (2017)

28. H. Kang, Y. Zhu, X. Yang, J. Shen, C. Chen, C. Li, Gold/ mesoporous silica-fiber core-shell hybrid nanostructure: a potential 
electron transfer mediator in a bio-electrochemical system. New J. Chem. 34, 2166 (2010)

29. G. Ortega-Zarzosa, J.R. Martínez, A. Robledo-Cabrera, G.A. Martínez-Castañón, M.G. Sánchez-Loredo, F. Ruiz, Annealing Behavior of Silica Gel Powders Modified with Silver Crystalline Aggregates. J. Sol-Gel Sci. Technol. 27, 255-262 (2003)

30. K. Sneha, A. Esterle, N. Sharma, S. Sahi, Yucca-derived synthesis of gold nanomaterial and their catalytic potential. Nanoscale Res. Lett. 9, 627 (2014)

31. H. Mundoor, S. Srivastava, Tunable variation of optical properties of polymer capped gold nanoparticles. J. Basu, Eur. Phys. J. D 49, (2008)

32. A. Quintanilla, V.C.L. Butselaar-Orthlieb, C. Kwakernaak, W.G. Sloof, M.T. Kreutzer, F. Kapteijn, Weakly bound capping agents on gold nanoparticles in catalysis: Surface poison? J. Catal. 271, 104-114 (2010)

33. M. Grasemann, G. Laurenczy, Formic acid as a hydrogen source recent developments and future trends. Energy Environ. Sci. 5, $8171(2012)$

34. Q.-Y. Bi, X.-L. Du, Y.-M. Liu, Y. Cao, H.-Y. He, K.-N. Fan, Efficient Subnanometric Gold-Catalyzed Hydrogen Generation via Formic Acid Decomposition under Ambient Conditions. J. Am. Chem. Soc. 134, 8926-8933 (2012)

35. D.A.K.R. Police, M. Chennaiahgari, R. Boddula, S.V.P. Vattikuti, K.K. Mandari, C. Byon, Single-step hydrothermal synthesis of wrinkled graphene wrapped $\mathrm{TiO} 2$ nanotubes for photocatalytic hydrogen production and supercapacitor applications. Mater. Res. Bull. 98, 314-321 (2018)

36. R. S. Coffey, The decomposition of formic acid catalysed by soluble metal complexes. Chem. Commun. 18, 923b-924 (1967). https://doi.org/10.1039/C1967000923B

37. Y. Gao, J. K. Kuncheria, H. A. Jenkins, R. J. Puddephatt, G. P. A. Yap, The interconversion of formic acid and hydrogen/carbon dioxide using a binuclear ruthenium complex catalyst. J. Chem. Soc., Dalton Trans. 18, 3212-3217 (2000). https://doi.org/10.1039/ B004234J

38. Y. Himeda, N. Onozawa-Komatsuzaki, H. Sugihara, H. Arakawa, K. Kasuga, Half-Sandwich Complexes with 4,7-Dihydroxy-1,10phenanthroline: Water-Soluble, Highly Efficient Catalysts for Hydrogenation of Bicarbonate Attributable to the Generation of an Oxyanion on the Catalyst Ligand. Organometallics 23, 1480 $1483(2004)$

39. A. Boddien, D. Mellmann, F. Gaertner, R. Jackstell, H. Junge, P.J. Dyson, G. Laurenczy, R. Ludwig, M. Beller, Efficient Dehydrogenation of Formic Acid Using an Iron Catalyst. Science 333, 1733-1736 (2011)

40. Y. Himeda, Highly efficient hydrogen evolution by decomposition of formic acid using an iridium catalyst with 4,4'-dihydroxy-2,2'bipyridine. Green Chem. 11, 2018-2022 (2009)

41. X. Zhou, Y. Huang, W. Xing, C. Liu, J. Liao, T. Lu, High-quality hydrogen from the catalyzed decomposition of formic acid by $\mathrm{Pd}-$ $\mathrm{Au} / \mathrm{C}$ and $\mathrm{Pd}-\mathrm{Ag} / \mathrm{C}$. Chem. Commun. 30, 3540-3542 (2008)

42. D.A. Bulushev, S. Beloshapkin, J.R.H. Ross, Hydrogen from formic acid decomposition over Pd and Au catalysts. Catal. Today 154, 7-12 (2010)

43. K. Tedsree, T. Li, Hydrogen production from formic acid decomposition at room temperature using a $\mathrm{Ag}-\mathrm{Pd}$ core-shell nanocatalyst. Nat. Nanotechnol. 6, 302-307 (2011)

44. M. Ojeda, E. Iglesia, Formic Acid Dehydrogenation on Au-Based Catalysts at Near-Ambient Temperatures. Angew. Chem. Int. Ed. 48, 4800-4803 (2009)

45. D. Preti, S. Squarcialupi, G. Fachinetti, Production of $\mathrm{HCOOH} /$ $\mathrm{NEt}_{3}$ Adducts by $\mathrm{CO}_{2} / \mathrm{H}_{2}$ Incorporation into Neat $\mathrm{NEt}_{3}$. Angew. Chem. Int. Ed. 49, 2581-2584 (2010)

46. P. Brack, S. Dann, K. G. U. Wijayantha, P. Adcock, S. Foster, A Simple, Low-cost, and Robust System to Measure the Volume of Hydrogen Evolved by Chemical Reactions with Aqueous Solutions. JoVE J. Vis. Exp. e54383 (2016)

47. L. Liu, A. Corma, Evolution of Isolated Atoms and Clusters in Catalysis. Trends Chem. 2, 383-400 (2020)

48. M. A. Asoro, P. J. Ferreira, D. Kovar, In situ transmission electron microscopy and scanning transmission electron microscopy studies of sintering of Ag and Pt nanoparticles. Acta Mater. 81, 173-183 (2014)

49. D. Nasrallah M, Sintering process and catalysis. Int. J. Nanomater. Nanotechnol. Nanomedicine 001 (2018)

50. N. Zanganeh, V.K. Guda, H. Toghiani, J.M. Keith, Sinter-Resistant and Highly Active Sub-5 nm Bimetallic $\mathrm{Au}-\mathrm{Cu}$ Nanoparticle Catalysts Encapsulated in Silica for High-Temperature Carbon Monoxide Oxidation. ACS Appl. Mater. Interfaces 10, 4776$4785(2018)$ 